\title{
Effects of D-002 on Formaldehyde-Induced Osteoarthritis in Rats
}

\author{
Sarahi Mendoza, Miriam Noa, Maikel Valle, Nilda Mendoza, Rosa Mas. \\ Center of Natural Products/ National Center for Scientific Researchs
}

\begin{abstract}
:
BACKGROUND: The treatment of Osteoarthritis $(O A)$ is addressed to pain alleviation and functional status improvement. Non-steroidal anti-inflammatory drug (NSAIDs), widely prescribed for OA, have been show to produce gastrointestinal (non selective) and cardiovascular (ciclooxygenase- 2 inhibitors) adverse effects. Then, the search for new substances is justified. D-002 is a mixture of higher aliphatic beeswax alcohols with anti-inflammatory and gastroprotective, rather than gastrotoxic effects.
\end{abstract}

OBJECTIVE: To investigate the effects of D-002 on formaldehyde-induced OA in rats.

METHODS: OA was induced by formaldehyde injection. Animals were orally dosed with either D-002 (50, 200 and $400 \mathrm{mg} / \mathrm{kg}$ ) or naproxen (NAP) $(3 \mathrm{mg} / \mathrm{kg})$, as reference drug. Controls comprised negative and vehicletreated rats. Treatments were administered for 10 days. The effects were assessed by measuring the changes on the diameters of rat ankle and paw.

RESULTS: The formaldehyde injection significantly increased the diameters of rat paw and ankle as compared to the negative control group, changes that were significantly decreased by naproxen. All doses of D-002 significantly reduced these increases as compared to the positive control, but not dose-dependently. The effects of the lowest and highest doses were practically the same. The effect of the highest dose of D-002 was significantly lower than that of NAP.

CONCLUSIONS: Oral administration of D-002 (50 - $400 \mathrm{mg} / \mathrm{kg})$ significantly decreased the formaldehydeinduced increases of rat paw and ankle enlargement, albeit less effectively than NAP. These results encourage to investigate its effects on other experimental models of $O A$.

KEYWORDS:Osteoarthritis, Beeswax, D-002, NSAIDs, formaldehyde.

\section{INTRODUCTION}

Osteoarthritis (OA), a common clinical syndrome characterized by joint pain, often associated to joint rigidity, mainly in the morning, leads to a progressive reduction of joint function that impair the quality of life of sufferers, mainly the elderly [1-3]. OA mostly affect the weight-bearing joints, like the knees, hips, cervical, lumbar and sacral spine, and feet, but also the interphalangeal joints of hand fingers [1-4]. The high prevalence of OA supports significant costs to the society, particularly relevant for elderly persons who face potential loss of independence. It is expected that these costs increase as the life expectancy does $[2,4]$.

The typical joint degeneration linked to OA involves mechanical and biochemical events that disrupt the balance between the degradation and synthesis of the joint cartilage in favor of the former, thus causing the destruction of joint tissue, particularly the synovial membrane and subchondral bone. Although OA is thought to be mainly due to excessive wear and tear, secondary inflammatory changes may also affect the joints [5-7].

The aims of OA treatment include alleviate the pain and improve the functional status of the subjects. Non-pharmacologic approaches, including weight loss, systematic exercise, patient education, physical and occupational therapy, joint unloading, among others, are key pieces of OA treatment $[1,4,8,9]$. Nevertheless, often they alone do not control properly the symptoms and pharmacological intervention is needed.Pharmacological therapy of OA includes use acetaminophen for mild or moderate pain without obvious inflammation; which switches to non-steroidal anti-inflammatory drug (NSAIDs) if the clinical response to acetaminophen is unsatisfactory or if the clinical presentation is mainly inflammatory [10,11]. NSAIDs, commonly used to treat inflammation and associated pain, represent the most prescribed drugs to treat OA [12], but their use is hampered by their adverse side effects, mainly gastrointestinal (non selective NSAIDs) and cardiovascular (ciclooxygenase -COX- 2 inhibitors) adverse effects [13-16] Then, the search for new antiinflammatory substances is justified. 
Besides, intra-joint corticosteroid injection and viscosupplementation may alleviate the pain and inflammation of the affected joint [3,17]. None of these therapies, however, is focused in repair the loss of the cartilage, for which intake of big amounts of glucosamine and/or chondroitin sulfate have been used, as they tend replace the damaged cartilage, although not all results have been uniformly positive [18-20]. Arthroplasty is indicated if all these modalities are ineffective, or if patients optimally treated cannot perform their usual activities [3].

D-002, a mixture of six higher aliphatic primary alcohols (C24, C26 C28, C30, C32, C34) purified from beeswax wherein triacontanol (C30) is the most abundant [21], has been shown anti-inflammatory effects in models of acute and chronic inflammation [22-24]. Differently from NSAIDs gastrotoxicity, however, D-002 treatment produce gastroprotective effects through a mechanism that involves increased secretion and improved composition of the gastric mucus [25-30], and reduced lipid peroxidation [28,30]. The aim of this study was to investigate the effects of D-002 on formaldehyde-induced OA in rats, a simple experimental model of OA $[31,32]$.

\subsection{ANIMALS}

\section{MATERIALS AND METHODS}

Male Wistar rats (190- $230 \mathrm{~g})$ from the National Centre for Laboratory Animal Production (CENPALAB) (Havana, Cuba) were adapted to laboratory conditions for seven days $\left(25 \pm 2{ }^{\circ} \mathrm{C}\right.$, humidity $60 \pm$ $10 \%$, light/darkness cycles of 12 hours), with food and water provided ad libitum. Animal care and handling were in accordance with the Principles of Laboratory Animal Care and the Cuban Regulations for the use of laboratory animals. The study protocol was consistent with our approved Standard Operational Procedures.

\subsection{SUBSTANCES}

The batch of D-002 used in the experiment was supplied by Plants of Natural Products (Havana, Cuba) after confirm its quality specifications. The composition of the batch was assessed with a validated gas chromatography method [21]. For administration, D-002 was suspended in 2\% Tween 20/ $\mathrm{H}_{2} \mathrm{O}$ vehicle. Naproxen (QUIMEFA Pharmaceutical Industry, Havana, Cuba) was dissolved in sodium bicarbonate 5\%.

\subsection{TREATMENTS}

Animals were distributed into 6 groups of 8 animals each: a negative vehicle control and five groups that received formaldehyde injection: one positive control, treated orally with the vehicle, three treated with D-002 (50, 200 and $400 \mathrm{mg} / \mathrm{kg})$, and other with naproxen $(3 \mathrm{mg} / \mathrm{kg})$ (NAP), the reference drug. All treatments (vehicle, D-002, naproxen) were administered once daily by gastric gavage $(5 \mathrm{~mL} / \mathrm{kg}$ ) for 10 days.

\subsection{Formaldehyde-induced arthritis test}

On day one, rats of all groups, except the negative control, were administered with the respective treatments (vehicle, D-002, NAP). One hour later, arthritis was induced by injecting $0.1 \mathrm{~mL}$ of $2.0 \%$ formaldehyde solution into the right hind, and this procedure was repeated on day 3 .

The diameter of the paw and that of the right ankle were measured on day one, before the induction of the damage, and after 10 days on treatment. Measurements were done by determining the perimeter, using a tied thread around the surface and a graded rule. The anti-arthritic activity, assesses as the anti- inflammatory response, was calculated as follows: $\%=(1-\mathrm{T} / \mathrm{C}) \times 100$, where $\mathrm{T}$ and $\mathrm{C}$ are the mean diameters of the treated and positive control groups, respectively $[33,34]$.

\subsection{Statistical analysis}

Comparisons were done with the Wilcoxon test for matched samples (within group comparisons) and the Mann Whitney U test (between group comparisons). The level of statistical significance was chosen at $\alpha=$ 0.05. Data were processed with the Statistic software package for Windows (Release 6.1, StatSoft Inc, Tulsa, OK, USA).

\section{RESULTS AND DISCUSSION}

The injection of formaldehyde significantly increased the diameters of rat paw and ankle as compared to the negative control group (Table 1), changes that were significantly decreased by naproxen, the reference drug. These results, consistent with those of other authors [33,34], confirm the validity of the model in our experimental conditions, and then the effects of the D-002 treatment here described. All doses of D-002 (50 $400 \mathrm{mg} / \mathrm{kg}$ ) significantly reduced the formaldehyde-induced increases of rat paws as compared to the positive control (26.9 - $30.8 \%$ ) and baseline, but not dose-dependently (Table 1). The effects of the lowest and highest doses were practically the same, and significantly lower than that of NAP (46.1\% inhibition). The effects of 
D-002 and NAP on the ankle were greater than on the paw enlargement. In this case, the maximal reduction of the edematous response with D-002 (51.7\%), obviously greater than that of $50 \mathrm{mg} / \mathrm{kg}(44.8 \%)$, was reached with $200 \mathrm{mg} / \mathrm{kg}$, being similar to that of $400 \mathrm{mg} / \mathrm{kg}$. The effect of the highest dose of D-002 was significantly lower than that of NAP $(89.7 \%)$.

These results are consistent with the anti-inflammatory effects of D-002 in models of acute (carrageenan-induced pleurisy in rats, xylene-induced mouse ear edema), and chronic (cotton pellet granuloma) inflammation [22-24], and expand the evidences of its anti-inflammatory effects on chronic inflammation in a model that, involving specifically the inflammation on rat ankle and paw, mimics some key features of OA [31,32].

The formaldehyde-induced model of arthritis, useful to assess the potential anti-arthritic and antiinflammatory effects of a substance, partially resembles the characteristics of human arthritis [3134].Formaldehyde injection causes a chronic inflammation of the rat foot, which involves the proliferation phase of inflammation elicited by COX mediators [31-34]. Then, it is logical that naproxen, a non selective NSAIDs that inhibits both COX-1 and COX-2 enzyme isoforms, effectively reduced the formaldehyde-induce increases in the size of the rat paw and ankle, particularly on this last one (46.1 and 89.7\%, respectively). The effects of D-002 $(50-400 \mathrm{mg} / \mathrm{kg})$ on this model, although clearly effective, were modest as compared to those of naproxen. The difference in the effectiveness of both treatments should be explained on terms of their respective mechanisms, but the mechanism of the anti-inflammatory effect of D-002 has not been reported. Nevertheless, keeping in mind that D-002 is devoid of gastrotoxicity, it should not inhibit the COX-1 isoform, which is highly present in the gastroduodenal mucosa $[14,15]$. In addition, it should be remembered that leukotrienes (LT), products formed in the lipooxygenase (LOX) pathway, play an important role in increasing the vascular permeability and the consequent edema formation in the exudation, but not in the proliferation phase of inflammation that characterizes this model. Keeping in mind that D-002 significantly reduced the concentrations of $\mathrm{LTB}_{4}$ in the pleural exudates of rats with carrageenan-induced pleurisy [22], an inhibition of LOX, rather than of COX activity, could be mainly involved in the effects of D-002, which could explain the present results.

\section{CONCLUSIONS}

Oral administration of D-002 $(50-400 \mathrm{mg} / \mathrm{kg})$ significantly decreased the formaldehyde-induced increases of rat paw and ankle enlargement, albeit less effectively than NAP. These results are consistent with previous reports of the anti-inflammatory effects of D-002, and encourage to investigate its effects on other experimental models of OA.

\section{REFERENCES}

[1] D.L. Hunter and D.T. Nelson, Osteoarthritis, BMJ, 332, 2006, 639-642

[2] S.V. Garstang and T.P. Stitik, Osteoarthritis: epidemiology, risk factors, and pathophysiology, Am J Phys Med Rehabil, 85, 2006, S2-S11.

[3] W. Zhang, R.W. Moskowitz, G. Nuki, S. Abramson, R.D. Altman, N. Arden, et al, OARSI recommendations for the management of hip and knee osteoarthritis. Part II: OARSI evidence-based expert consensus guidelines, Osteoarthritis Cartilage, 16, 2008, 137-162.

[4] C. Lozada and E. Steigelfest, Osteoarthritis. Medicine Rheumatology, 15, 2010, 12-23.

[5] J. Martel-Pelletier, Pathophysiology of osteoarthritis. Osteoarthritis Cartilage, 12 (Suppl A), 2004, S31-S33.

[6] M.B. Goldring and S.R. Goldring, Osteoarthritis. J Cell Physiol, 213, 2007, 626-634.

[7] J. Johanne and J.P. Pelletier, Is osteoarthritis a disease involving only cartilage or other articular tissues?, Eklem Hastalık Cerrahisi, 21, 2010, 2-14.

[8] E. Roddy and M. Doherty, Changing life-styles and osteoarthritis: what is the evidence?, Best Pract. Res. Clin Reumatol, 20, 2006, 81-97.

[9] Y.C. Lee and R.H. Shmerling, The benefit of non-pharmacologic therapy to treat symptomatic osteoarthritis. Curr Rheumatol Rep, 10, 2008, 5-10.

[10] T.E. Towheed, L. Maxwell, M.G. Judd, M. Catton, M.C. Hochberg and G. Wells, Acetaminophen for osteoarthritis. Cochrane Database Syst Rev, 25, 2006, CD004257.

[11] B. Bannwarth, Acetaminophen or NSAIDs for the treatment of osteoarthritis, Best Pract Res Clin Rheumatol, 20, 2006, 117-129.

[12] C.R. Scanzello, N.K. Moskowitz and A. Gibofsky, The post-NSAID era: what to use now for the pharmacologic treatment of pain and inflammation in osteoarthritis, Curr Rheumatol Rep, 10, 2008, 49-56.

[13] S.S. Mikhail, N.A. Zwar, S. Vagholkar, S.M. Dennis and R.O. Day, Non-steroidal anti-inflammatory drugs in general practice: a decision-making dilemma, Med J Aust, 187, 2007, 160-163.

[14] D. Lamarque, Pathogenesis of gastroduodenal lesions induced by non-steroidal anti-inflammatory drugs, Gastroenterol Clin Biol, 28, 2004, C18-26.

[15] B.J. Whittle, Gastrointestinal effects of non-steroidal anti-inflammatory drugs, Fundam Clin Pharmacol, 17, $2003,301-313$.

[16] R.A. Moore, S. Derry and H.J. McQuay, Cyclo-oxygenase-2 selective inhibitors and non-steroidal anti-inflammatory drugs: balancing gastrointestinal and cardiovascular risk, BMC Musculoskelet Disord, 8, 2007, 73-77.

[17] E.J. Strauss, J.A. Hart, M.D. Miller, R.D. Altman and J.E. Rosen, Hyaluronic acid viscosupplementation and osteoarthritis: current uses and future directions. Am J Sports Med, 37, 2009, 1636-1644.

[18] K. Pavelka, J. Gatterova, M. Olejarova, S. Machacek, G. Giacovelli and C. Rovatil, Glucosamine sulphate use and delay of progression of knee osteoarthritis: a 3-year randomized placebo-controlled double-blind study, Arch Intern Med, 162, 2002, 2113-2123. 
T. McAlindon, Why are clinical trials of glucosamine no longer uniformly positive?, Rheum Dis Clin North Am, 29, 2003, 789801.

E.C. Huskisson, Glucosamine and chondroitin for osteoarthritis, J Int Med Res, 36, 2008, 1161-1179.

V. González, D. Marrero, R. Sierra, C. Velázquez and R. Vicente, Nuevo método por Cromatografía Gaseosa Capilar para el análisis del ingrediente activo D002, Revista CENIC Cien Quím, 39, 2008, 23-124.

D. Carbajal, V. Molina, S. Valdés, M.L. Arruzazabala, R. Más and J. Magraner, Anti-inflammatory activity of D-002: an active product isolated from beeswax, Prostagl Leukotr Essent Fatty Acids, 59, 1998, 235-238.

Y. Ravelo, V. Molina, D. Carbajal, M.L. Arruzazabala, R. Mas, A. Oyarzábal, et al, Effects of single oral and topical administration of D-002 (beeswax alcohols) on xylene induced ear edema in mice, LAJP, 29, 2010, 1451-1454.

Y. Ravelo, V. Molina, D. Carbajal, L. Fernández, J. Fernández, M.L. Arruzazabala, et al, Evaluation of anti-inflammatory and antinociceptive effects of Abexol (beeswax alcohols), J Nat Med, 65, 2010, 330-335.

D. Carbajal, V. Molina, S. Valdés, M.L. Arruzazabala and R. Mas, Anti-ulcer activity of higher primary alcohols of beeswax, $J$ Pharm Phamacol, 47, 1995, 731-733.

D. Carbajal, V. Molina, S. Valdés, M.L. Arruzazabala, I. Rodeiro, R. Mas, et al, Possible cytoprotective mechanism in rats of D002 an anti-ulcerogenic product isolated from beeswax, J Pharm Pharmacol, 48, 1996, 858-860.

D. Carbajal, V. Molina, M. Noa, S. Valdes, M.L. Arruzazabala, C. Aguiar, et al. Effects of D-002 on gastric mucus composition in ethanol-induced ulcer, Pharmacol Res, 42, 2000, 329-332.

V. Molina, S. Valdés, D. Carbajal, M.L. Arruzazabala, R. Menéndez and R. Más, Antioxidant effects of D-002 on gastric mucosa of rats with experimentally-induced injury, J Med Food, 4, 2001, 79-83.

J. Illnait, H. Terry, R. Mas, L. Fernandez and D. Carbajal, Effects of D-002 a product isolated from beeswax on gastric symptoms of patients with osteoarthritis treated with piroxicam: a pilot study, J Med Food, 8, 2005, 63-68.

E. López, J. Illnait, V. Molina, A. Oyarzábal, L. Fernández, Y. Pérez, et al, Effects of D-002 (Beeswax Alcohols) on Lipid Peroxidation in Middle-aged and Older Subjects, LAJP, 27, 2008, 695-703.

M. Bendele, Animal models of rheumatoid arthritis, J Musculoskel Neuron Interact, 1, 2001, 377-385.

R.A. Greenwald, Animal models for evaluation of arthritic drugs, Method Find Clin Pharmacol, 13, 1991, 75-83.

S. Singh and D.K. Majumdar, Effects of fixed oil of Ocimum sanctum against experimentally induced arthritis and joint edema in laboratory animals, Pharmaceutical Biology, 34, 1996, 218-222.

C.O. Okoli, P.A. Akah, N.J. Onuoha, T.C. Okoye, A.C. Nwoye and Ch.S. Nworu, Acanthus montanus: An experimental evaluation of the antimicrobial anti-inflammatory and immunological properties of a traditional remedy for furuncles. $B M C$ Complement Altern Med, 8, 2008, 27.

Table 1. Effects of D-002 on the rat paw and ankle diameters $(\mathrm{X} \pm \mathrm{SD})(\mathrm{mm})$

\begin{tabular}{|c|c|c|c|}
\hline Treatments & Baseline & 10 days & $\begin{array}{c}\text { Inhibition } \\
(\%)\end{array}$ \\
\hline \multicolumn{4}{|c|}{ Effects on the rat paw diameter } \\
\hline Negative vehicle control & $24.2 \pm 1.4$ & $24.2 \pm 1.4^{+++}$ & - \\
\hline Positive control (formaldehyde + vehicle) & $25.0 \pm 0.0$ & $26.8 \pm 0.7^{* *}$ & - \\
\hline Formaldehyde $+\mathrm{D}-002(50 \mathrm{mg} / \mathrm{kg})$ & $24.6 \pm 0.5$ & $26.1 \pm 0.6^{+* *}$ & 26.9 \\
\hline Formaldehyde +D-002 (200 mg/kg) & $24.7 \pm 0.5$ & $26.0 \pm 0.8^{+*}$ & 30.8 \\
\hline Formaldehyde +D-002 $(400 \mathrm{mg} / \mathrm{kg})$ & $24.7 \pm 0.5$ & $26.1 \pm 0.7^{+* *}$ & 26.9 \\
\hline Formaldehyde +naproxen $3 \mathrm{mg} / \mathrm{kg}$ & $24.8 \pm 0.4$ & $25.6 \pm 0.5^{++^{*}}$ & 46.1 \\
\hline \multicolumn{4}{|c|}{ Effects on the rat ankle diameter } \\
\hline Negative vehicle control & $20.3 \pm 0.71$ & $20.3 \pm 0.71^{+++}$ & - \\
\hline Positive control (formaldehyde + vehicle) & $21.5 \pm 1.35$ & $23.2 \pm 0.92^{*}$ & - \\
\hline Formaldehyde $+\mathrm{D}-002(50 \mathrm{mg} / \mathrm{kg})$ & $20.2 \pm 1.32$ & $21.9 \pm 1.3^{*}$ & 44.8 \\
\hline Formaldehyde +D-002 (200 mg/kg) & $20.7 \pm 0.82$ & $21.7 \pm 1.1^{*++}$ & 51.7 \\
\hline Formaldehyde +D-002 (400 mg/kg) & $20.8 \pm 1.32$ & $21.7 \pm 0.9^{*++}$ & 51.7 \\
\hline Formaldehyde +naproxen $3 \mathrm{mg} / \mathrm{kg}$ & $20.2 \pm 0.42$ & $20.6 \pm 0.7^{+++}$ & 89.7 \\
\hline
\end{tabular}

${ }^{*} \mathrm{p}<0.05 ;{ }^{* *} \mathrm{p}<0.01$. Comparisons with baseline (Wilcoxon test for matched samples) ${ }^{+} p<0.05 ;{ }^{++} p<0.01 ;{ }^{++} p<0.001$, Comparisons with positive control (Mann Whitney U test) 